\title{
Study on Flow Characteristic of Cold Fluid in Supersonic Combustion Experiment Table
}

\author{
LI Qiana, PAN Hub, DUAN Li-weic, DING Xiao-yu ${ }^{d}$ \\ State Key Laboratory of Laser Propulsion \& Application, Equipment Academy, Beijing 101416, \\ China \\ aliqian827@sohu.com, ${ }^{b}$ panhuu@gmail.com, cduanliweidlw@126.com, dingxiaoyu001@163.com
}

Keywords: Supersonic combustion, cold fluid flow, flow characteristic, numerical simulation, TDLAS

\begin{abstract}
Flow characteristic of cold fluid in supersonic combustion experiment table is studied through the method of computational fluid dynamics (CFD) numerical simulation, direct measurement of pressure transducer and TDLAS measurement. Characteristic parameters of temperature and pressure etc. of the flow field are obtained respectively under four work conditions, under which the total temperature and pressure of inflow is $782 \mathrm{~K}$ and $7.2 \mathrm{~atm}, 814 \mathrm{~K}$ and $6.3 \mathrm{~atm}$, $850 \mathrm{~K}$ and $6.2 \mathrm{~atm}, 912 \mathrm{~K}$ and $6.0 \mathrm{~atm}$. Results indicate that computational pressure of CFD numerical simulation adopting SIMPLE algorithm and $k-\varepsilon$ Realizable turbulence model is consistent with that of direct pressure measurement on each measurement position. However, since under the experimental condition, air heater combustion generated triatomic molecule, which would make the specific heat ratio lower than that of ideal gas model used in CFD numerical simulation, so the flow velocity is lower in experiments, but the pressure is higher. Temperature and pressure obtained from TDLAS measurement agree well with the analog simulation according to CFD results. Temperature deviation of the front and rear of experimental segment is $4.2 \%$ and $10 \%$, while the pressure deviation is $5.3 \%$ and $1.4 \%$ respectively. It can be seen that TDLAS would be applied in fluid parameters' measurement of supersonic combustion well as a kind of non-contact flow field diagnostic techniques.
\end{abstract}

\section{Introduction}

Traditional temperature and mass flow measuring method is the adoption of wall sensor and intrusion probe ${ }^{[1,2]}$. With study of combustion field continually deepening, more limitation appears on traditional measuring method ${ }^{[3]}$. Thermocouple inside probe needs complex thermal conduction model for measuring temperature accurately ${ }^{[4]}$. Besides, fluid flow is usually disturbed by probe in combustion field, and unacceptable shocks will appear ${ }^{[5]}$. Therefore, non-contact laser measurement technology becomes the certain choice. Laser measurement technology includes scattering ${ }^{[6]}$, fluorescence $^{[7]}$, interference ${ }^{[8]}$ and absorption ${ }^{[5]}$, etc, among which tunable diode laser absorption spectroscopy (TDLAS) technology is relatively mature and shows extensive prospect ${ }^{[9,10]}$. Fluid field parameters, such as temperature, component concentration, velocity, mass flow, momentum and thrust can be obtained by TDLAS technology, which is helpful to increase efficiency, reduce pollutant emission, and so on. Aimed at the situation that combustion chamber of supersonic combustion experiment table not being ignited, which is called cold fluid flow in this paper, flow characteristic is studied through numerical simulation, contact measurement and TDLAS measuring method. Accuracy of numerical and experimental method is validated, and powerful support is provided for study on flow characteristic after combustion chamber being ignited.

\section{Computational Method}

\section{Computational model}

Governing equations are 2D unsteady compressible Reynolds average N-S equations. SIMPLE algorithm with first order precision based on pressure, density and momentum is adopted. The 
turbulence model is $k-\varepsilon$ Realizable. Turbulent viscosity coefficient of $k-\varepsilon$ Realizable model is the same as that of standard $k-\varepsilon$ model, while the difference is that $C_{\mu}$ is not constant.

\section{Initial and boundary conditions}

All parameters on far field boundary are fixed as that of the inflow because the inflow is supersonic. Outflow boundary is pressure outflow, on which pressure is $10000 \mathrm{~Pa}$ and temperature is 200K. Wall is adiabatic no-slip condition. Upstream of the isolated segment is fixed a nozzle of Mach 2, which is used to simulate flight enthalpy of Mach 5. Four work conditions are selected through changing total temperature and pressure, which are shown in Table 1.

Table 1 Experimental work conditions

\begin{tabular}{ccc}
\hline Work condition & Total temperature/K & Total pressure/atm \\
\hline 1 & 782 & 7.2 \\
2 & 814 & 6.3 \\
3 & 850 & 6.2 \\
4 & 912 & 6.0 \\
\hline
\end{tabular}

\section{Experiment System and Test Method}

\section{Supersonic combustion experiment table}

Experiment table is composed of air supply system, air heating system, steady segment, nozzle, experimental segment and air exhaust system. The photograph of experimental segment is shown in Fig. 1. Experimental segment is columnar, whose diameter is $200 \mathrm{~mm}$, height is $600 \mathrm{~mm}$. Molded lines of the nozzle is Mach 2.5, area $30 \mathrm{~mm} \times 20 \mathrm{~mm}$. 10 pressure tapping holes are assembled on the underside wall of experimental segment to measure static pressure. The photograph of pressure transmitters are shown in Fig. 2.

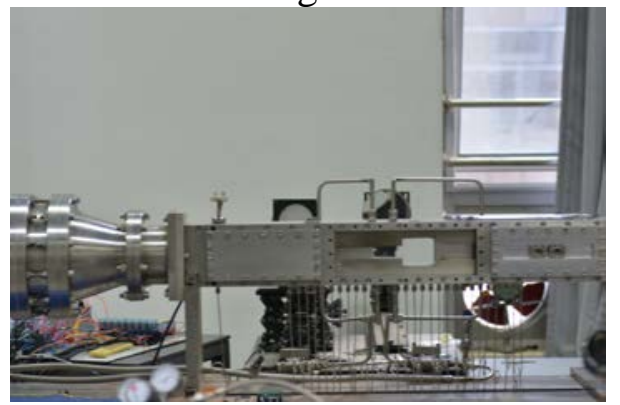

Fig. 1 Photograph of experimental segment

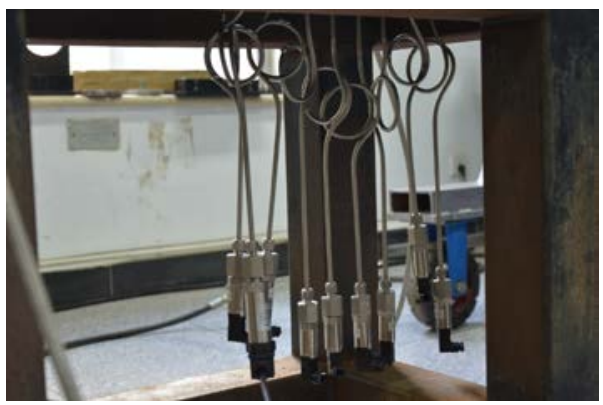

Fig. 2 Photograph of pressure transducer

\section{TDLAS measuring method}

$\mathrm{H}_{2} \mathrm{O}$ is regarded as object molecule. According to the measured environment, following the requirement that absorption line intensity $>10^{-4} \mathrm{~cm}^{-2} \mathrm{~atm}^{-1}$, a couple of absorption spectrum is selected among 1340 1470nm from HITRAN2008 data base.

Laser output by two fiber coupling DBD lasers(wave length $\lambda_{1}=1391.7 \mathrm{~nm}, \lambda_{2}=1388.1 \mathrm{~nm}$ ) is coupled into one fiber by polarization-preserving fiber coupler, and then comes into fiber beam splitter. Ninety percent of channel is received by InGaAs electrophotonic detector after it is emitted by collimating lens, so the transfer function of time vs. frequency while laser is scanning can be confirmed. Ten percent of channel is connected with fiber beam splitter and split into two beams with the same intensity The two beams are directed into test region by polarization-preserving single mode fiber. After they are emitted with collimation, they pass through wedge window and drill through the flow field upward and downward. Beams drilled through the fluid are captured by large diameter coupling lens, and then enter into electrophotonic detector and data acquisition system through multimode fiber transmitting. The data acquisition system can simulate signal output, so it is used as modulation signal generator of laser. Nitrogen is used to purify all the optical path so as to avoid the interference on measurement by vapor in air. 


\section{Results and Discussion}

Curves of the pressure of the underside wall along axis $x$ under four work conditions are shown in Fig. 3. From Fig. 3, it can be concluded that numerical simulated results drive to that of direct measurement on each test point consistently. However, pressure in front of the combustion chamber measured in experiment is a little higher. Ideal gas state equation is adopted in numerical simulation, while the specific heat ratio is 1.4 , but in experiment, triatmoic molecules such as $\mathrm{H}_{2} \mathrm{O}$ and $\mathrm{CO}_{2}$ will generate with air heater combustion, while the specific heat ratio is between 1.33 and 1.4, that is to say, sound speed will descend, which will result in the descending of flow velocity, so the pressure value is higher than that of numerical simulation.

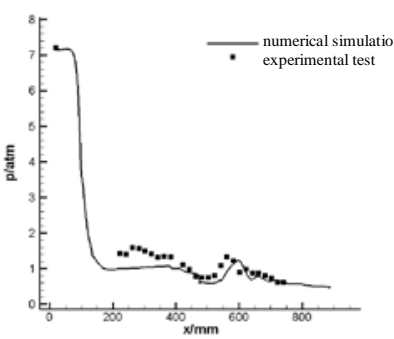

(a)work condition 1

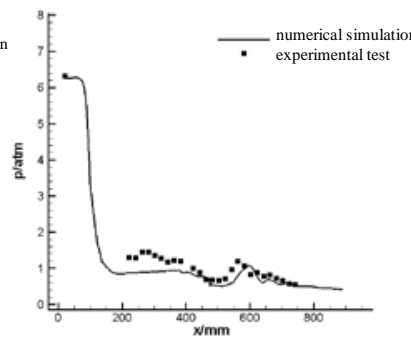

(b)work condition 2

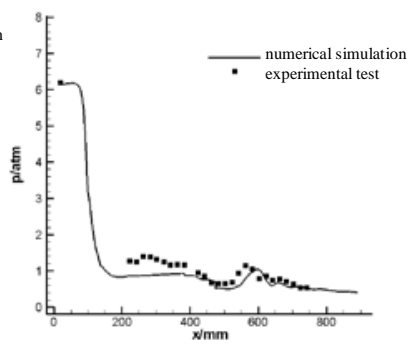

(c)work condition 3

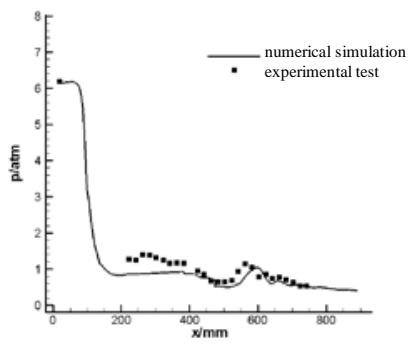

(d)work condition 4

Fig. 3 Pressure of the underside wall derived from numerical simulation vs. direct measurement by pressure transducer

Analog simulation is performed on TDLAS measuring method under the 3rd work condition(total temperature and pressure is $850 \mathrm{~K}$ and 6.2atm respectively). Analog simulation here refers to that according to pressure, temperature and velocity obtained from CFD, photoelectric signal of TDLAS measurement is obtained, and then pressure and temperature can be resolved in response to the signal. In the simulation, the concentration of $\mathrm{H}_{2} \mathrm{O}$ molecule is $10 \%$. The measuring layout is: two beams drill through the fluid field; each beam contains two-frequency laser, so the average temperature and pressure can be obtained individually on the path. Average is not path-average, but weighted average looking concentration of $\mathrm{H}_{2} \mathrm{O}$ molecule as the weight.

Results derived from three methods agree well, but the temperature is higher and pressure is lower measured by TDLAS. The specific difference is shown in Table 2. Percentage of deviation between emulation and test is expressed as -err., which is the ratio of the difference of the two results to measurement result. From Table 2 it can be seen that the pressure deviation is the smallest.

Table 2 Result of analog simulation vs. experimental measurement

\begin{tabular}{ccccccc}
\hline Measurement position & T-Sim. & T-Meas. & T-err. & P-Sim. & P-Meas. & P-err. \\
\hline Front of experimental segment & 427 & 445.5 & $4.2 \%$ & 0.476 & 0.452 & $5.3 \%$ \\
Rear of experimental segment & 412 & 458 & $10 \%$ & 0.422 & 0.428 & $1.4 \%$ \\
\hline
\end{tabular}

\section{Conclusion}

Flow characteristic of cold fluid in supersonic combustion experiment table under four work conditions is studied. The methods are CFD numerical simulation, direct measurement of pressure transducer and TDLAS measurement. Results indicate:

(1)Flow process of cold fluid in supersonic combustion can be simulated by SIMPLE algorithm and $k-\varepsilon$ Realizable turbulence model. Pressure values obtained from computation agree well with that obtained from direct measurement of pressure transducer.

(2)TDLAS measuring method, as a non-contact optical diagnostic technique, is very suitable for measuring temperature, pressure and component concentration of the fluid field.

(3)TDLAS measuring results agree well with that of analog simulation according to CFD results. Temperature deviation of the rear of experimental segment is $10 \%$, which is the largest, while the smallest is $1.4 \%$, which is the pressure deviation of the rear of experimental segment. 


\section{References}

[1] Miller M F, Kessler W J and Allen M G. Diode laser-based air mass flux sensor for subsonic aero-propulsion inlets[J]. Applied Optics, 1996, 35(24): 4905-4912.

[2] Williams S, Barone D, Barhorst T, et al. Diode laser diagnostics of high speed flows[R]. AIAA 2006-7999.

[3] Williams J G, Steenken W G, Yuhas A J. Estimating engine air-flow in gas-turbine powered aircraft with clean and distorted inlet flows[R]. NASA CR-198052, 1996.

[4] Albertson C W, Bauserman W A. Total temperature probes for high-temperature hypersonic boundary-layer measurements[R]. NASA Tech, Mem 4407, 1993.

[5] Upschulte B L, Miller M F, Allen M G. Diode laser sensor for gasdynamic measurements in a model scramjet combustor[J]. AIAA Journal, 2000, 38(7): 1246-1252.

[6] Varghese P L, Phadke C and Fink M. A novel Raman technique for flow diagnostics[R]. AIAA 1996-0176.

[7] McKenzie R L. Progress in laser spectroscopic techniques for aerodynamic measurements: an overview[J]. AIAA Journal, 1993, 32(3): 465-477.

[8] Otugen M, Ganguly B. Laser heterodyne method for high-resolution gas-density measurements[J]. Applied Optics, 2001, 40(21):3502-3505.

[9] Allen M G. Diode laser absorption sensors for gas-dynamic and combustion flows[J]. Measurement Science and Technology, 1998, 9: 545-562.

[10] Tao Bo, Hu Zhiyun, Wang Sheng, et al. Study on the measurement of combustion temperature based on TDLAS technique. Journal of Engineering Thermophysics, 2013, 35(2): 401-404. 